\title{
UNSTEADY MULTIPHASE MODELING OF CAVITATION AROUND NACA 0015
}

\author{
Abolfazl Asnaghi \\ Department of Mechanical Engineering, Sharif University of Technology, Iran \\ Ebrahim Jahanbakhsh \\ Department of Mechanical Engineering, Sharif University of Technology, Iran. \\ Mohammad Saeed Seif \\ Department of Mechanical Engineering, Sharif University of Technology, Iran., seif@sharif.edu
}

Follow this and additional works at: https://jmstt.ntou.edu.tw/journal

Part of the Engineering Commons

\section{Recommended Citation}

Asnaghi, Abolfazl; Jahanbakhsh, Ebrahim; and Seif, Mohammad Saeed (2010) "UNSTEADY MULTIPHASE MODELING OF CAVITATION AROUND NACA 0015," Journal of Marine Science and Technology. Vol. 18: Iss. 5, Article 9.

DOI: 10.51400/2709-6998.1915

Available at: https://jmstt.ntou.edu.tw/journal/vol18/iss5/9

This Research Article is brought to you for free and open access by Journal of Marine Science and Technology. It has been accepted for inclusion in Journal of Marine Science and Technology by an authorized editor of Journal of Marine Science and Technology. 


\title{
UNSTEADY MULTIPHASE MODELING OF CAVITATION AROUND NACA 0015
}

\author{
Abolfazl Asnaghi*, Ebrahim Jahanbakhsh*, and Mohammad Saeed Seif*
}

Key words: cavitation, unsteady flow, numerical simulation, NACA 0015 .

\begin{abstract}
The present study focuses on the numerical simulation of cavitation around the NACA 0015. The unsteady behaviors of cavitation which have worthwhile applications are investigated. The cavitation patterns, velocity fields and frequency of the cavitating flow around hydrofoil is obtained. For multi phase simulation, single-fluid Navier-Stokes equations, along with a volume fraction transport equation, are employed. The bubble dynamics model is utilized to simulate phase change. SIMPLE algorithm is used for velocity and pressure computations. For discretization of equations the finite-volume approach written in body fitted curvilinear coordinates, on collocated grid, is used. In this study, unsteady simulation of cavitation around NACA 0015 is investigated. Nevertheless the obtained results can be extended for other geometry with considering related factors such as the effects of cavitation number, the effect of scale, and the constants in the cavitation model.

In what follows, the cavitation model and governing equations are briefly summarized. Following this, derivation of numerical algorithm is presented to explain modeling concepts. The results cover simulation of flow over a NACA 0015 in cavitating and non-cavitating situations. The frequency of flow, pressure distributions, tip cavitation effects and the cavitating vortex shedding are the main results that are analyzed.
\end{abstract}

\section{INTRODUCTION}

When local static pressure of a liquid falls below the corresponding saturated pressure, the phase of fluid changes from liquid to vapor. This phenomenon is named cavitation. The cavitation is departure from evaporation. The evaporation, in definition, is performed by temperature changing but cavitation is performed by pressure changing. Cavitation phenomenon can be observed in a wide variety of propulsion and power

Paper submitted 07/27/09; revised 08/27/09; accepted 09/15/09. Author for correspondence: Mohammad Saeed Seif (e-mail: seif@sharif.edu).

*Department of Mechanical Engineering, Sharif University of Technology, Iran. systems like pumps, nozzles and injectors.

Cavitation is categorized by a dimensionless number that called cavitation number, where it depends on the vapor pressure, the liquid density, the main flow pressure and the main flow velocity, respectively. When cavitation number of flow reduces the probability of cavitation formation increases. Usually the cavitation formation of a flow is categorized based on the cavitation number of the flow.

In a flow over the body, five different cavitation regimes are observed: incipient, shear, cloud, partial, and super-cavitation. Undesirable aspects of cavitation phenomenon are erosion, structural damages, noise and power loss in addition to beneficial features such as drag reduction and the effects of cavitation in water jet washing systems. The drag reduction observed on bodies surrounded fully or partially with cavity strongly encourages one to research on the cavitating flows.

As aforementioned, apart from the damage associated with cavitation, there are applications in which one can benefit from cavitation. The vaporous cavity surrounding the vehicle causes many problems related to control and guidance. The most important reasons are Vortex shedding, bubble collapse and phase change between liquid and vapor. Therefore, to design a perfect control system and to prevent from dislike aspects of cavitation, accurate and detailed information of the flow field is required. In addition to experimental investigations of cavitation, computational fluid dynamics, CFD, can provide useful tools which using them can help one to know about whole details of cavitation flows.

Using computational models for cavitation has been around for the last few decades. Early works mainly used potential flow theory. Complex characteristics of cavitating flows such as sharp changes in the fluid density, existence of a moving boundary and the requirement of modeling phase change forced the development of computational algorithms based on Navier-Stokes equations. Following the advancement in CFD methods, cavitation models based on Navier-Stokes equations emerged in early 1990's. Among these models, the two main categories are interface tracking method and homogeneous equilibrium flow. In the first category, a constant pressure (vapor pressure) is assumed for the cavity region (the so-called cavity) and a wake model is used to predict the shape of the cavity in adaptive grids. The current study is based on the second category, where a single-fluid modeling approach is employed for both phases. 
Homogeneous equilibrium flow model assumes that there is no velocity slip between the phases at the cavity interface. A common approach for evaluating the density is the application of barotropic water-vapor state laws. Usually selection of appropriate state law is a difficult task which requires enough experimental data from a specific problem. Additionally, a barotropic state equation ignores vorticity production at the cavity closure, which is an important characteristic of cavitating flows.

A more precise and applicable approach to solve an equation for liquid (or vapor) volume fraction and compute density is the volume fraction method. This approach, so-named transport based equation model (TEM), has widely been applied to simulate cavitation.

Two major points should be considered regarding TEM approach: 1) a solution strategy for advection equation, 2) the selection of an appropriate mass transfer model. Yuan et al. [14] suggested a cavitation model based on Rayleigh equation. Singhal et al. [13], Merkle et al. [6] and Kunz et al. [5] used different mass transfer models based on semi-analytical equations. Senocak and Shyy [11, 12] recommended a completely analytical cavitation model based on local mass-momentum transfer around the cavity interface.

In this study the bubble dynamic model which is based on the Rayleigh equation is used. For solving the governing equations a SIMPLE algorithm is employed in a finite volume approach to solve the fluid flow. A finite-volume approach written in body fitted curvilinear coordinates, on collocated grids in $2 \mathrm{D}$ and $3 \mathrm{D}$ domains is used for the numerical discretization.

For the present study, the transport equation-based model, described earlier, is implemented into the solver and related modifications, regarding the convection schemes and the SIMPLE algorithm, have been made for time-dependent computations.

The main objective of this study is simulation of cavitation around NACA 0015. Based on this simulation, the pressure distribution, the drag and lift coefficients and the frequency of cavitation are obtained. The interaction between cavitation and flow around the hydrofoil, cavitation vortex shedding and its effects are analyzed. Finally, the obtained results are compared with experimental results to demonstrate the accuracy of the current simulation.

\section{GOVERNING EQUATIONS}

\section{The Volume Fraction Function}

In multiphase flow the location of any fluid is specified using a volume fraction function. For example in vapor-liquid two phase flow, $\alpha_{v}=1$ denotes inside the vapor and $\alpha_{v}=0$ in the liquid. Cells which lie between 0 and 1 contain the interface. This mentioned concept can be written as:

$$
\alpha_{v}(x, t)=\left\{\begin{array}{cc}
0 & \text { Liquid Phase } \\
0<\alpha_{v}<1 & \text { Liquid-Vapor } \\
1 & \text { Vapor Phase }
\end{array}\right.
$$

\section{The Homogenous Model}

With determination of the volume of fraction, the local properties of fluid can be achieved base on the single state of each phase. This method is named the homogenous model. The mixture density and viscosity are defined as follows based on the vapor volume fraction:

$$
\begin{aligned}
& \rho_{m}=\alpha_{v} \rho_{v}+\left(1-\alpha_{v}\right) \rho_{l} \\
& \mu_{m}=\alpha_{v} \mu_{v}+\left(1-\alpha_{v}\right) \mu_{l}
\end{aligned}
$$

\section{The Homogenous Model}

The equations governing the flow of a compressible fluid are the continuity equation, the momentum equations, and the energy equation. This set of non-linear, coupled equations is solved for the unknowns $\rho, v, T$ and $P$. In index notation form, these equations may be written as:

$$
\begin{gathered}
\frac{\partial \rho_{m}}{\partial t}+\frac{\partial\left(\rho_{m} u_{i}\right)}{\partial x_{i}}=0 \\
\frac{D\left(\rho_{m} u_{i}\right)}{D t}=-\frac{\partial(P)}{\partial x_{i}}+\frac{\partial}{\partial x_{j}}\left(\mu\left(\frac{\partial u_{i}}{\partial x_{j}}+\frac{\partial u_{j}}{\partial x_{i}}\right)\right)+\rho_{m} g_{i} \\
\frac{D\left(\rho_{m} T\right)}{D t}=\frac{1}{C_{P}} \frac{\partial}{\partial x_{j}}\left(K \frac{\partial T}{\partial x_{j}}\right)+\frac{\beta T}{C_{P}}\left[\frac{D(P)}{D t}\right]+\frac{(\varphi+\dddot{q})}{C_{P}}
\end{gathered}
$$

Where $\varphi$ denotes the dissipation term in energy equation and $\beta$ is the thermal expansion coefficient which is equal to $1 / \mathrm{T}$ for an ideal gas. In addition to the above differential equations, an auxiliary equation of state relating density to pressure and temperature $(\rho=f(P, T))$ is needed. For an ideal gas, this equation is given by:

$$
\rho_{m}=\frac{P}{R_{m} T}=C_{\rho} P
$$

In many practical problems related to cavitation phenomena, the change in temperatures is negligible. Therefore the simulation of cavitation in isothermal condition has not any effect on final results. Therefore the pressure-density coupling is problematic and requires special attention.

As aforementioned, in this study the TEM is employed. With this equation, pressure and density are connected implicitly. To simulate phase change between vapor and liquid, a term, $S_{\alpha}$, is added to the right side of vapor volume fraction equation.

$$
\frac{\partial \alpha_{v}}{\partial t}+\frac{\partial\left(\alpha_{v} u_{i}\right)}{\partial x_{i}}=S_{\alpha}
$$


For solving the conservation of mass equation two main approaches are exist. One is conservative approach and the other is non-conservative approach. In the conservative approach, the effect of mixture density gradient is hidden in the relation and when this type of equation is solved the effect of mixture density gradient is considered implicitly. But in the non-conservative approach the non-zero velocity divergence is used (Kunz et al. [5]). In this part we demonstrate the relation between the volume fraction equation source term and the considered source term in the velocity divergence equation. With substituting the mixture density relation, Eq. (2), in the mass conservation equation, Eq. (3), it can be obtained:

$$
\begin{gathered}
\frac{\partial \alpha}{\partial t}+\frac{\partial\left(\alpha u_{i}\right)}{\partial x_{i}}=S_{\alpha} \\
\frac{\partial u_{i}}{\partial x_{i}}=S_{m} \\
S_{m}=\frac{\rho_{l}-\rho_{v}}{\rho_{l}} S_{\alpha} \rightarrow\left(\rho_{v}<<\rho_{l}\right) \rightarrow S_{m}=S_{\alpha}
\end{gathered}
$$

The above equation presents the relation between the volume fraction equation source term and the source term in the velocity divergence equation. In the present study, the nonconservative form of the mass conservation equation is utilized and effects of the velocity divergence source term are assumed to make relation between pressure and density.

\section{DISCRETIZATION METHOD}

\section{General Scheme}

A finite volume method is used to discretize the equations. The details related to the discretization methods of the Navier Stokes equations and the conservation of mass equation can be found in [12].

In the discretized form of Navier-Stokes equations there are three major terms: unsteadiness, convection and diffusion. The discretization of the diffusion flux does not require any special consideration and the method adopted here is the second order estimation. The discretization of the convection flux is, however, problematic and requires special attention. In this study, the Upwind scheme is used to calculate the faces values. For the representation of the unsteady term, the gridpoint value of $\varphi$ is assumed to prevail throughout the control volume and the time derivative is approximated using an Euler-implicit formulation [2, 4].

To discretize the volume of fraction transport equation it is necessary to compute the values on the interfaces accurately. For capturing shock it is necessary to use high order methods such as HRIC [3, 9]. Because of the low speed flow, The upwind method is used to calculate the volume fraction values on computational cells faces. With homogeneous approach and volume fraction values in faces, the density on the cell interface can be calculated.

\section{Volume of Fraction Discretization}

With application the finite volume approach, and Euler implicit method the discretized form of volume of fraction equation can be found as:

$$
\alpha_{P}^{t+\delta t} \frac{V_{P}}{\delta t}+\sum_{f=1}^{n}\left(\alpha_{f}^{t+\delta t} F_{f}\right)=S_{\alpha}+\alpha_{P}^{t} \frac{V_{P}}{\delta t}
$$

In this equation, fluxes and the source term are computed with the previous time step values.

\section{Velocity-Pressure-Density Algorithm}

For solving the N. S. and continuity discretized algebraic equations, the SIMPLE algorithm is employed. The major problematic challenge is creation of smooth connection among volume fraction equation, the Navier-Stokes equations and pressure correction equation [8].

The pressure correction equation is derived from mass continuity equation. First we consider mass continuity equation in non-conservative format:

$$
\frac{\partial\left(u_{i}\right)}{\partial x_{i}}=S_{m}
$$

With definitions of correction method we can mention that the corrected velocity domain consists of momentum velocity results and corrective terms that must be calculated from the pressure correction equation.

$$
u_{i}=u_{i}^{*}+u_{i}^{\prime}
$$

Substituting the above equation in the non-conservative from of mass continuity equation, it is obtained that:

$$
\frac{\partial\left(u_{i}^{*}+u_{i}^{\prime}\right)}{\partial x_{i}}=S_{m} \rightarrow \frac{\partial\left(u_{i}^{\prime}\right)}{\partial x_{i}}=S_{m}-\frac{\partial\left(u_{i}^{*}\right)}{\partial x_{i}}
$$

In the SIMPLE algorithm, corrective terms are obtained from Navier-Stokes equations, using Mokhalled and Darvish formats [7], It is obtained that:

$$
u_{i}^{\prime}=-D_{P}\left(\frac{\partial P^{\prime}}{\partial x_{i}}\right), \text { where: } D_{P}=\frac{\text { Volume }_{\text {Cell }}}{a_{P}}
$$

The pressure correction equation can be achieved with combination the Eqs. (12) and (13): 


$$
\begin{aligned}
\frac{\partial}{\partial x_{i}}\left[-D_{P}\left(\frac{\partial P^{\prime}}{\partial x_{i}}\right)\right] & =S_{m}-\frac{\partial\left(u_{i}^{*}\right)}{\partial x_{i}} \\
& \rightarrow D_{P} \frac{\partial}{\partial x_{i}}\left[\left(\frac{\partial P^{\prime}}{\partial x_{i}}\right)\right]=\frac{\partial\left(u_{i}^{*}\right)}{\partial x_{i}}-S_{m}
\end{aligned}
$$

In the cavitating flow the pressure correction equation plays important rule. When we use the non-conservative format, we add a relative big term to the source term of algebraic pressure correction equation. This term may cause instability in solving procedure. One of the best ways to overcome this instability is using the fully implicit approach in solving all equations. For keeping in implicit form, we must correct $S_{m}$ to keep all terms in the new time step. This approach is suggested by Sauer [10]. With using the first order Tyler series for $S_{m}$ around pressure of previous time step or iteration, it is obtained that:

$$
\begin{gathered}
\left.S_{m}\right|_{\text {new }}=\left.S_{m}\right|_{\text {old }}+\frac{\partial}{\partial P}\left(\left.S_{m}\right|_{\text {old }}\right) \cdot P^{\prime} \\
\text { Where: } P^{\prime}=P_{\text {new }}-P_{\text {old }}
\end{gathered}
$$

Therefore the modified pressure correction equation is obtained as:

$$
D_{P} \frac{\partial}{\partial x_{i}}\left(\frac{\partial P^{\prime}}{\partial x_{i}}\right)+\frac{\partial}{\partial P}\left(\left.S_{m}\right|_{o l d}\right) \cdot P^{\prime}=\frac{\partial u_{i}^{*}}{\partial x_{i}}-\left.S_{m}\right|_{o l d}
$$

By this approach, unsuitable effects of cavitating source term are reduced, because its derivation is added to diagonal coefficient matrix. For each time step, first, the vapor fraction transport equation is solved and a new vapor fraction distribution is obtained. Consequently the values of the mixture density and viscosity are updated. Based on these new values, the source term for the pressure correction equation and its derivation are evaluated. The Navier-Stokes equations and the modified pressure correction equation are solved until a convergence criterion is reached. Then, the whole procedure is repeated within the next time step. For numerical simulation, the pressure level usually is defined by the pressure boundary condition at the outlet of the computational domain. The utilized algorithm is drawn in Fig. 1. Other tools such as turbulence modeling, and rigid body motion modeling can be easily add to this algorithm. Study of such flows is remained for further research.

\section{CAVITATION MODEL}

In TEM approach, numerical models of cavitation differ in cavitation source term. The cavitation source term can define

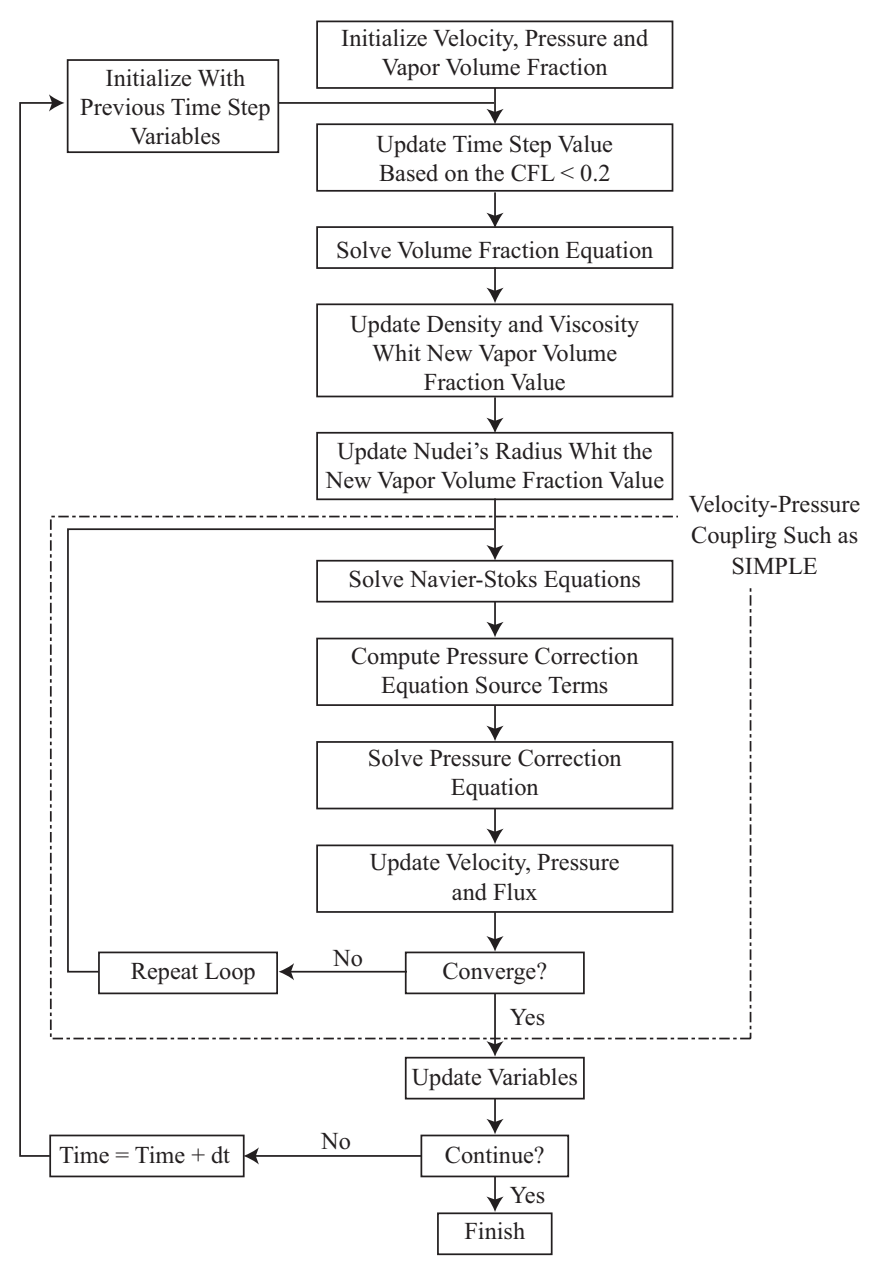

Fig. 1. The cavitation solver algorithm.

vapor net mass generation that contains effects of both vapor production and vapor destruction of the flow. In this study we consider the bubble dynamics method as the phase change model [2] which is presented by (17). In this study, the average nucleus per liquid volume is considered as $n_{0}=10^{8}$. Other properties such as the minimum radius of bubble can be calculated based on $n_{0}[10]$.

$$
\dot{m}=S_{m}=\operatorname{sign}\left(P_{\text {sat }}-P\right) \frac{n_{0}}{1+n_{0} \frac{4}{3} \pi R^{3}} 4 \pi R^{2} \sqrt{\frac{2\left|P_{\text {sat }}-P\right|}{3 \rho_{l}}}
$$

\section{NUMERICAL RESULTS}

\section{Geometry and Boundary Conditions}

A two dimensional NACA 0015 hydrofoil is shown in Fig. 2. In this figure, the geometry and boundary conditions of the current simulation are illustrated. The uniform flow condition of $\mathrm{U}_{\infty}=12 \mathrm{~m} / \mathrm{s}$ was used at the inlet boundary, and both the non-slip and the pressure outlet boundary condition were applied to the treatment of the hydrofoil boundaries. 


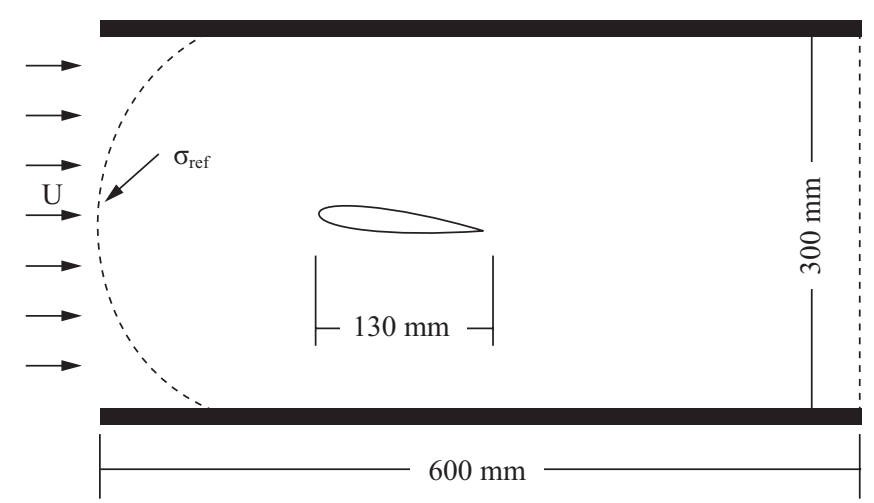

Fig. 2. The geometry and boundary conditions of 2D NACA.

Hydrofoil dimensions are shown in this figure.

In the current study the results are presented for the outlet cavitation numbers of one. The channel width is $300 \mathrm{~mm}$ and its length is $600 \mathrm{~mm}$. The chord length of the hydrofoil is 130 $\mathrm{mm}$ and the angle of attack is $6 \mathrm{deg}$.

The flow properties which are used in this study are presented in Table 1. Based on the homogenous theory, phases are considered as an incompressible fluid. By each phase's property, properties of the average fluid can be calculated.

\section{Non-Cavitation Simulation}

At first step, simulation of non-cavitation flows around NACA 0015 is done and compared with experimental result. With non-cavitating results, first, the accuracy of single phase simulation is investigated. Second, with comparison between cavitation and non-cavitation simulation, the effect of cavitation consideration in simulation can be demonstrated. The non-cavitation results are shone in the Fig. 3.

\section{The Unsteady Simulation}

The main objective of this study is to investigate the unsteady behavior of cavitation. The main parameters that are sought are the flow frequency and the cavitation patterns. It is obtained that with these implied boundary conditions, the pattern of flows repeats every 95 mile second. Therefore, the frequency of simulated flow is 10.53 hertz. To present the unsteady behavior of cavitation contours of vapor fraction for are shown in Fig. 4. The pattern of this flow can be categorized in five levels.

In the first level, time $=0$, there is no vapor around the hydrofoil. Due to fluctuations which generate from previous conditions, the pressure distribution is different from noncavitation conditions.

In the second level, time $=10 \mathrm{~ms}$ to $20 \mathrm{~ms}$, the tip cavitation occurs. In this condition, vapor covers tip of hydrofoil and initial vortex is created below the end of cavity. The interaction between cavity and vortex creation is shown in Fig. 5.

In the third level, time $=20 \mathrm{~ms}$ to $50 \mathrm{~ms}$, the reentrant jet at the back of vapor cavity is generated from initial vortex.
Table 1. The properties of liquid and vapor.

\begin{tabular}{|c|c|c|}
\hline Phase & Density $\mathrm{Kg} / \mathrm{m}^{3}$ & Viscosity $\mathrm{Kg} /(\mathrm{m} . \mathrm{s})$ \\
\hline Liquid & 1000 & $1 \times 10^{-3}$ \\
\hline Vapor & 1.0 & $1 \times 10^{-5}$ \\
\hline
\end{tabular}

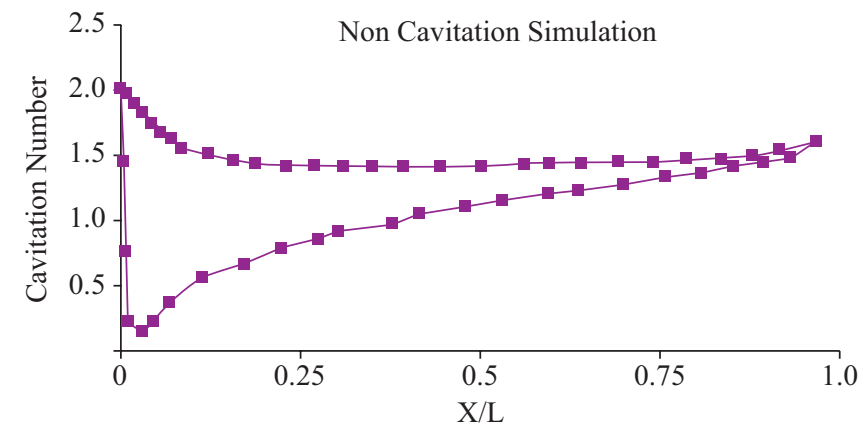

Fig. 3. Surface pressure distribution for non-cavitation flow.

This reentrant jet covers the surface between cavity and hydrofoil, Fig. 6. This reentrant jet eventually causes the cavity to separate from the hydrofoil surface. This reentrant jet eventually causes the cavity to separate from the hydrofoil surface.

In the fourth level, time $=50 \mathrm{~ms}$ to $90 \mathrm{~ms}$, the cavity is separated from hydrofoil surface completely. Because of flow pattern, around the end of the hydrofoil a main vortex is created. This vortex attracts the separated cavity. The main vortex around separated cavity is shown in Fig. 7. In this vortex, due to viscosity effects, pressure increases. When the separated cavity is attracted into the main vortex, because of higher pressure, phase change is occurred and vapor changes to liquid. Along with this phase change, the more little vortex is generated near the end of hydrofoil in the little vortex, due to generation of vortex, pressure decreases, and another vapor is generated in the end of hydrofoil. Finally, these two vortexes combine and all of vapor change into liquid. In addition to this process, near the middle of hydrofoil another vortex that doesn't contain any vapor is generated. These three vortex and their interactions with vapor phase are shown in Fig. 8.

In the fifth level, time $=90 \mathrm{~ms}$ to $95 \mathrm{~ms}$, there is no vapor, but due to fluctuations, the pressure distribution is different from non-cavitation flows.

\section{Validation}

For comparing presented numerical results with published experimental and numerical results, the Strohal number of the flow is calculated. The comparison between Strohal number of simulated flow and experimental results is shown in Fig. 9. According to the simulation parameters:

$$
\frac{\sigma}{2 \alpha}=4.775
$$

And, 


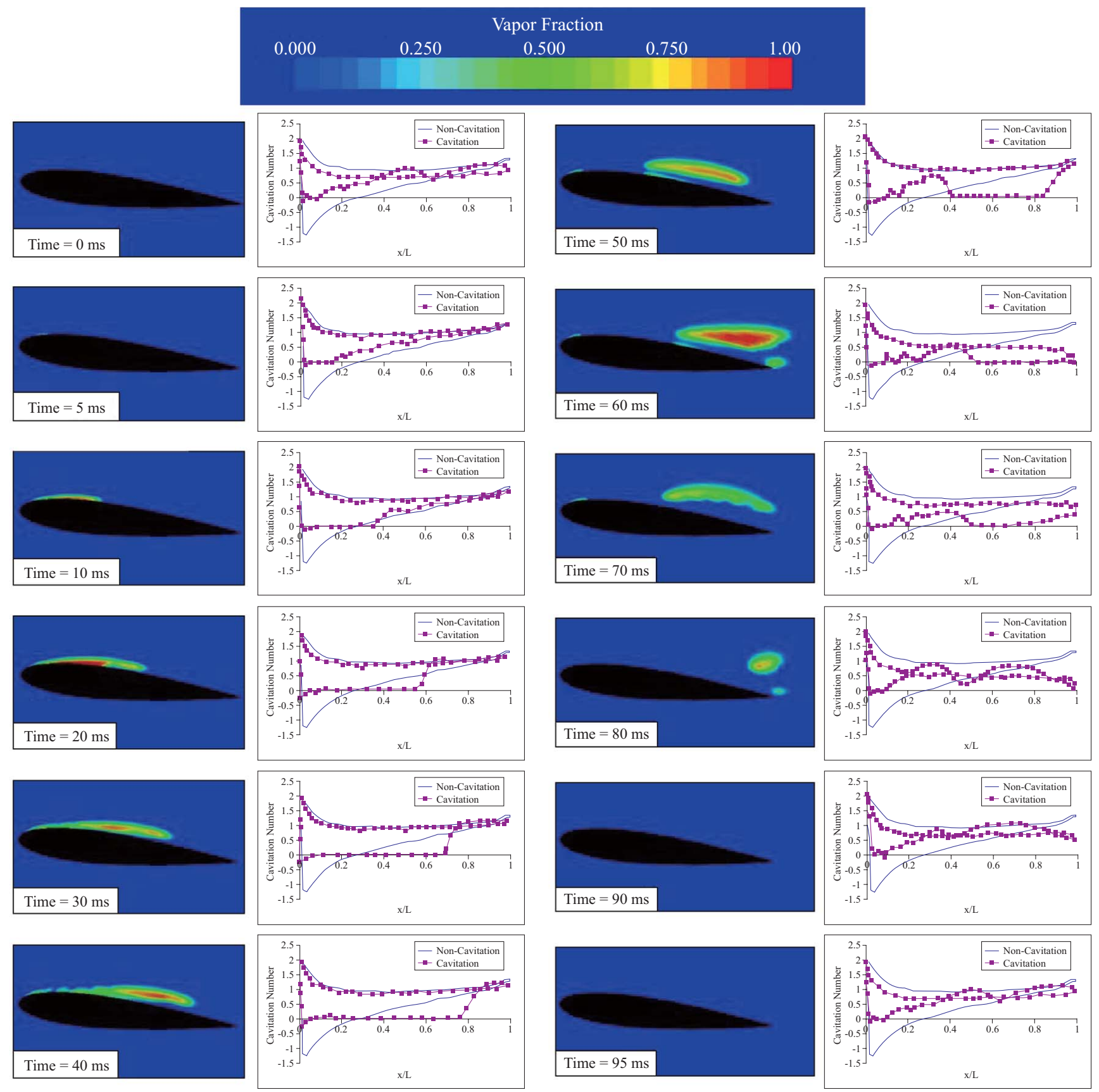

Fig. 4. Vapor volume fraction and pressure distribution over NACA 0015.

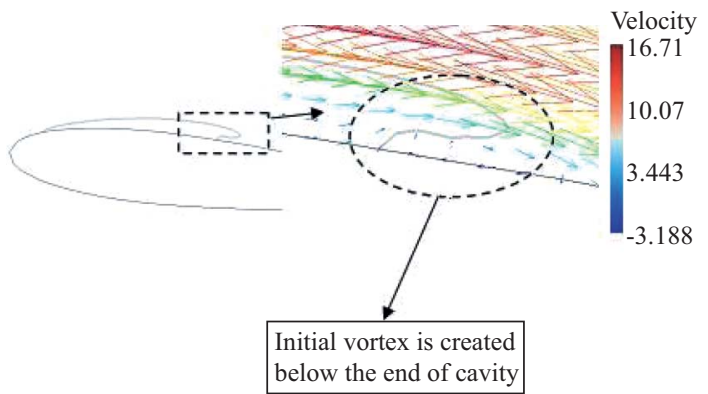

Fig. 5. The interaction between cavity and initial vortex (time $=15 \mathrm{e}-4)$.

$$
\begin{aligned}
& f=10.52 \mathrm{~Hz} \\
& C=0.8 \mathrm{~m} \\
& U=12 \frac{\mathrm{m}}{\mathrm{s}}
\end{aligned} \mid \quad S t=\frac{f C}{U}=0.7
$$

The maximum length of cavity can be obtained from Fig. 4 . In this figure, and in the time $=0.030 \mathrm{~ms}$, the maximum length of cavity is around 0.7 of the chord length. 


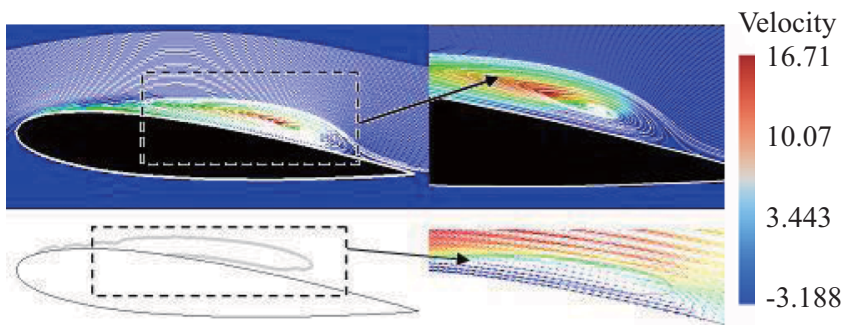

Fig. 6. The cavity separation due to created reentrant jet $($ time $=30 \mathrm{e}-4)$.

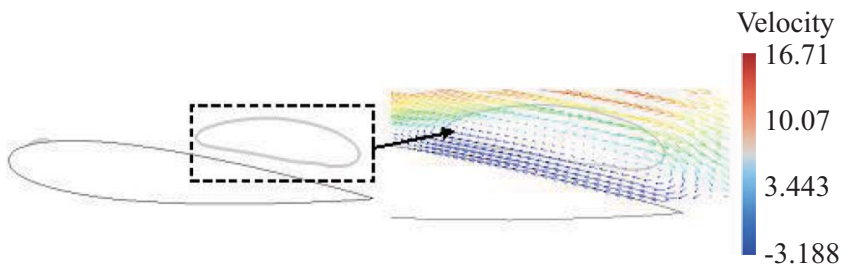

Fig. 7. Main vortex around the separated cavity $($ time $=55 \mathrm{e}-4)$.

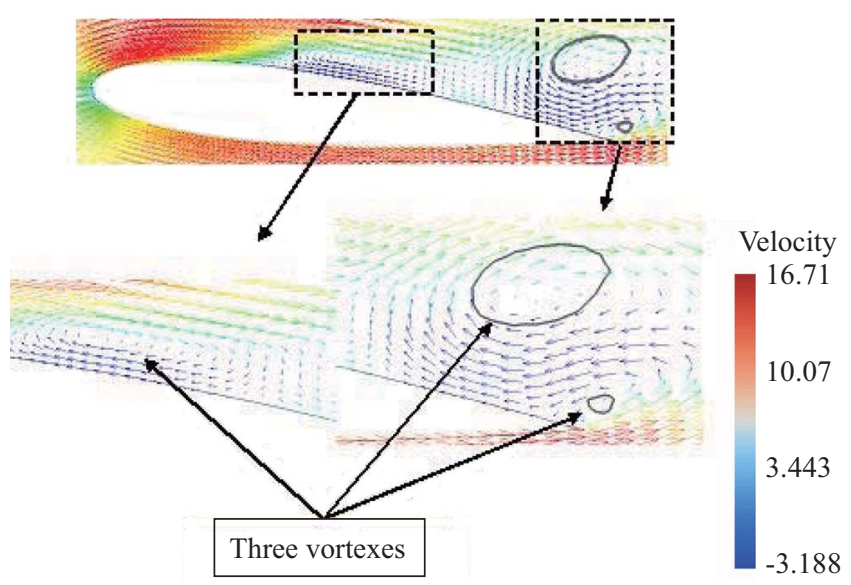

Fig. 8. Interaction of vapor volume fraction Iso-surface and three vortexes $($ time $=80 \mathrm{e}-3)$.

$$
\frac{l}{c}=0.7
$$

The comparison between non-dimensional cavity length and experimental results is shown in Fig. 10. This comparison shows a good agreement between presented results and experimental results.

\section{CONCLUSION}

A multi-phase numerical simulation is presented to simulate the cavitation phenomenon around NACA 0015. Different aspects of the analysis are demonstrated and an efficient and accurate simulation is acquired. The source terms in the volume of fluid equation and the continuity equation are calculated by the Bubble dynamics cavitation model. The SIMPLE

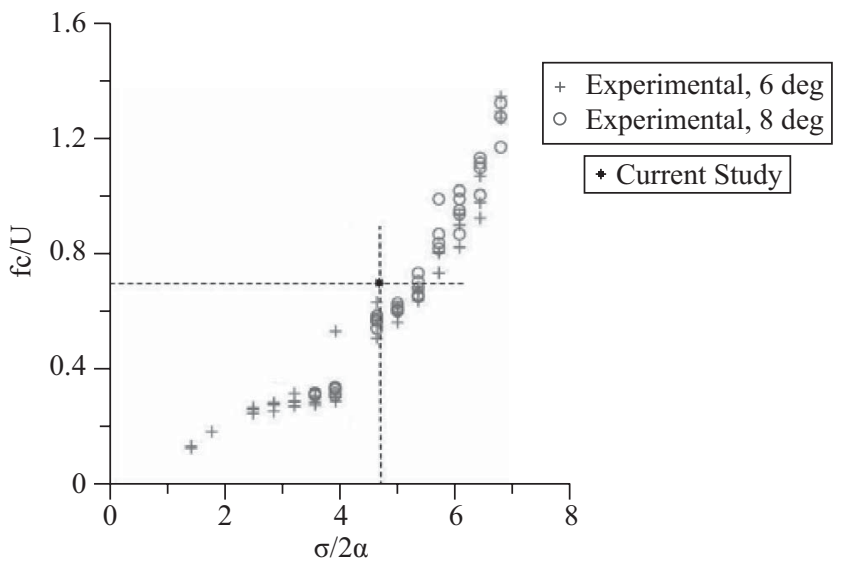

Fig. 9. Comparison between the observed Strohal number form numerical results and Published experimental data derived from reference [1] for NACA 0015.

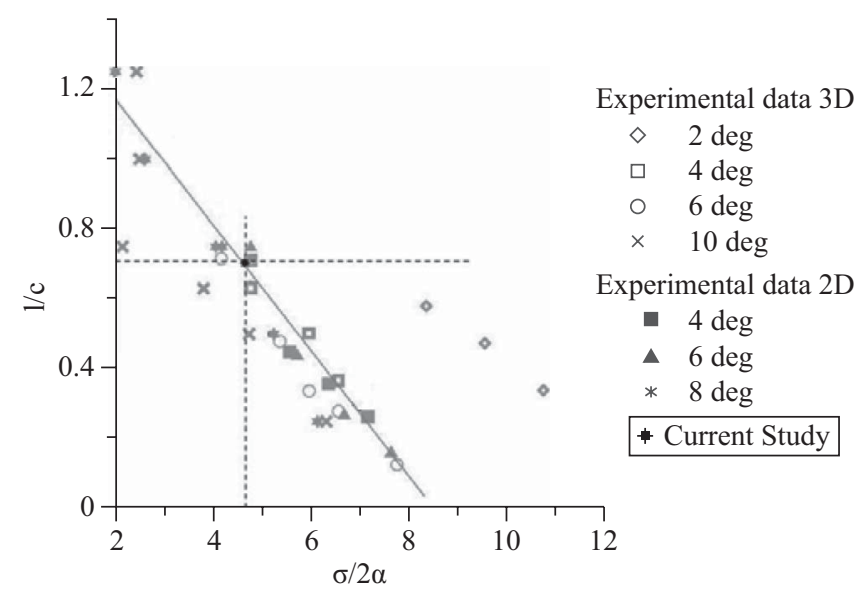

Fig. 10. Comparison between the Non-Dimensional cavity length and Published experimental data derived from reference [1] for NACA 0015.

method is used to solve coupling between the continuity and $\mathrm{N}$. S. equations.

The behavior of cavitation is classified into five levels. The inception of cavitation is detected in the tip of the hydrofoil. The initial generated bubbles aggrandize and develop along with chord of hydrofoil wall and make a vapor zone, called cavity. At the end of this cavity an initial vortex is created. Along with cavity growing, this vortex grows. Finally, this vortex causes the cavity to separate from hydrofoil surface. The separated cavity is transferred to the downstream area where due to the higher pressure they collapse. This process repeats with a specific frequency.

\section{NOMENCLATURE}

\section{Symbols}

$\dot{m} \quad$ Net vapor generation

$u_{i}, u, v, w \quad$ Cartesian velocity components 


\begin{tabular}{ll}
$x_{i}$ & Cartesian coordinates \\
$P$ & Pressure \\
$U$ & Velocity at a reference point \\
$f$ & Frequency \\
$C, L$ & Hydrofoil chord \\
$t$ & Time, mean flow time scale \\
$\alpha$ & Volume fraction of liquid \\
$\mu$ & Viscosity \\
$\rho$ & Density \\
$\sigma$ & Cavitation number \\
$g$ & Gravity vector \\
$S t$ & Strohal number \\
$l$ & Maximum length of cavity \\
$T$ & Temperature \\
$\delta t$ & Time step \\
$R$ & Nuclei radius \\
$n_{0}$ & Number of nucleus per volume \\
$C F L$ & Courant number \\
$F$ & Flux \\
$V$ & Volume \\
$S$ & Source term \\
$\Phi$ & Thermal dissipation \\
$\beta$ & Thermal expansion factor \\
$P^{\prime}$ & Pressure correction \\
$u^{\prime}$ & Velocity correction \\
$\frac{D}{D t}$ & Material derivation \\
$\frac{\partial}{\partial t}, \frac{\partial}{\partial p}, \frac{\partial}{\partial x}$ & Derivation's operators \\
\hline &
\end{tabular}

$\begin{array}{ll}\text { Subscripts, } & \text { Superscripts } \\ \infty & \text { Free stream } \\ i, j & \text { Coordinate indices } \\ m & \text { Mixture } \\ \text { sat } & \text { Saturation conditions } \\ l, v & \text { Liquid and vapor phase } \\ t & \text { Current time step } \\ t+\delta t & \text { Next time step }\end{array}$

\section{REFERENCES}

1. Berntsen, G. S., Kjeldsen, M., and Arndt, R. E. A., "Numerical modeling of sheet and tip vortex cavitation with fluent 5," Fourth International Symposium on Cavitation, California Institute of Technology, Pasadena, CA (2001).

2. Delannoy, Y. and Kueny, J. L., "Two-phase flow approach in unsteady cavitation modeling," Cavitation and Multiphase Flow Forum, New York, pp. 153-158 (1990).

3. Issa, R. I., "Solution of the implicitly discretized fluid flow equations by operator-splitting," Journal of Computational Physics, Vol. 62, pp. 40-65 (1985).

4. Jahanbakhsh, E., Panahi, R., and Seif, M. S., "Numerical simulation of three-dimensional interfacial flows," International Journal of Numerical Methods for Heat and Fluid Flow, Vol. 17, No. 4, pp. 384-404 (2007).

5. Kunz, R. F., Boger, D. A., Stinebring, D. R., Chyczewski, T. S., Lindau, J. W., Gibeling, H. J., Venkateswarn, S., and Govindan, T. R., "A preconditioned Navier-Stokes method for two-phase flows with application to cavitation prediction," Journal of Computers and Fluids, Vol. 29, pp. 849-875 (2000).

6. Merkle, C. L., Feng, J., and Buelow, P. E. O., "Computational modeling of the dynamics of sheet cavitation," Proceedings of 3rd International Symposium on Cavitation, Grenoble, France (1998).

7. Moukalled, F. and Darwish, M., "A unified formulation of the segregated class of algorithms for fluid flow at all speeds," Numerical Heat Transfer, Vol. 37, pp. 103-139 (2000).

8. Patankar, S. V. and Spalding, D. B., "A calculation procedure for heat, mass and momentum transfer in three dimensional parabolic flows," International Journal of Heat and Mass Transfer, Vol. 15, p. 1787 (1972).

9. Rhie, C. M. and Chow, W. L., "Numerical study of the turbulent flow past an airfoil with trailing edge separation," AIAA Journal, Vol. 21, pp. 15251532 (1983).

10. Sauer, J., Instationär Kavitierende Strömungen - Ein Neues Modell, Basierend auf Front Capturing (VoF) und Blasendynamik. Universität Karlsruhe, $\mathrm{Ph} . \mathrm{D}$. Thesis, Univeristy of Karlsruhe, Karlsruhe, Germany (2000).

11. Senocak, I. and Shyy, W., "Evaluation of cavitation models for NavierStokes computations," Proceedings of 2002 ASME Fluids Engineering Division Summer Meeting, Montreal, Canada (2002).

12. Senocak, I. and Shyy, W., "Numerical simulation of turbulent flows with sheet cavitation," Fourth International Symposium on Cavitation, California Institute of Technology, Pasadena, CA (2001).

13. Singhal, A. K., Vaidya, N., and Leonard, A. D., "Multidimensional simulation of cavitating flows using a PDF model for phase change," Proceedings of 1997 ASME Fluids Engineering Division Summer Meeting, Vancouver, p. 3272 (1997).

14. Yuan, W., Sauer, J., and Schnerr, G. H., "Modeling of unsteady cavitating flows in fuel injection nozzles," in: ILASS-Europe, Darmstadt, pp. 11-13 (2000). 